\title{
Application of Particle Swarm Optimization Algorithm in Test Points Selection of Radar Antenna Servo System
}

\author{
Han Ning ${ }^{1, a}$, Wu Guoqing ${ }^{2, b}$ Xia Mingfei ${ }^{3, c}$, Wang Xiaocong ${ }^{4, d}$, \\ Mechanical Technology Research Institute, Mechanical Engineering College Shijiazhuang, 050000, \\ China \\ aemail:haning1103@163.com
}

Keywords: particle swarm optimization, test points selection, radar system, antenna servo system

\begin{abstract}
To solve the problem that many test points are needed in radar antenna servo system when fault diagnosis is needed for this system, a new test points selection method based on Particle Swarm Optimization algorithm is researched. Test points selection is a many-target optimization problem which could easily get into local optimum value. To avoid this problem, target optimization function is constructed based on different fault modes and different fault position. The particles are constrained into a large area and the real-time particle update mode is used to make sure that the optimum value is not a local maximum or minimum value. The effectiveness of this method is validated by a simulation experiment.Equation Chapter 1 Section 1
\end{abstract}

\section{Introduction}

In precision tracking radar servo system, the main function is to control the antenna motion in various ways, the servo system performance directly affects the accuracy of radar measurement. Because the modern flight target speed and motor ability strengthens constantly, to achieve the fast acquisition and tracking of targets. Directly determine the target acquisition time radar servo system, the target tracking radar servo to ensure complete function. At the same time, the antenna to capture the target and tracking system with high performance, high speed and high reliability. Antenna control unit is the core of radar servo system, mainly responsible for the real-time data collection, field monitoring, real-time monitoring and management, antenna and PC communication tasks. High performance antenna control unit can realize high precision controllable radar servo system, high tracking accuracy for target.

In order to ensure the completeness of radar servo system has high real-time, need to repair to the servo system, repair the servo system of each test point by measuring the location error, but the radar servo system has complex structure, mechanical and electrical integration features, causes the test points, test is not convenient and long testing time and other problems, this led directly to the antenna servo system is unable to repair, which affect the combat effectiveness of radar. In order to solve the above problem, in this paper, the process of particle swarm optimization algorithm of selecting test points of test points, to optimize the use of particle swarm algorithm, in the prerequisite for high fault detection rate and fault false alarm rate, reduce diagnosis time, increase the rate of complete servo system. 


\section{The structure of radar servo system}

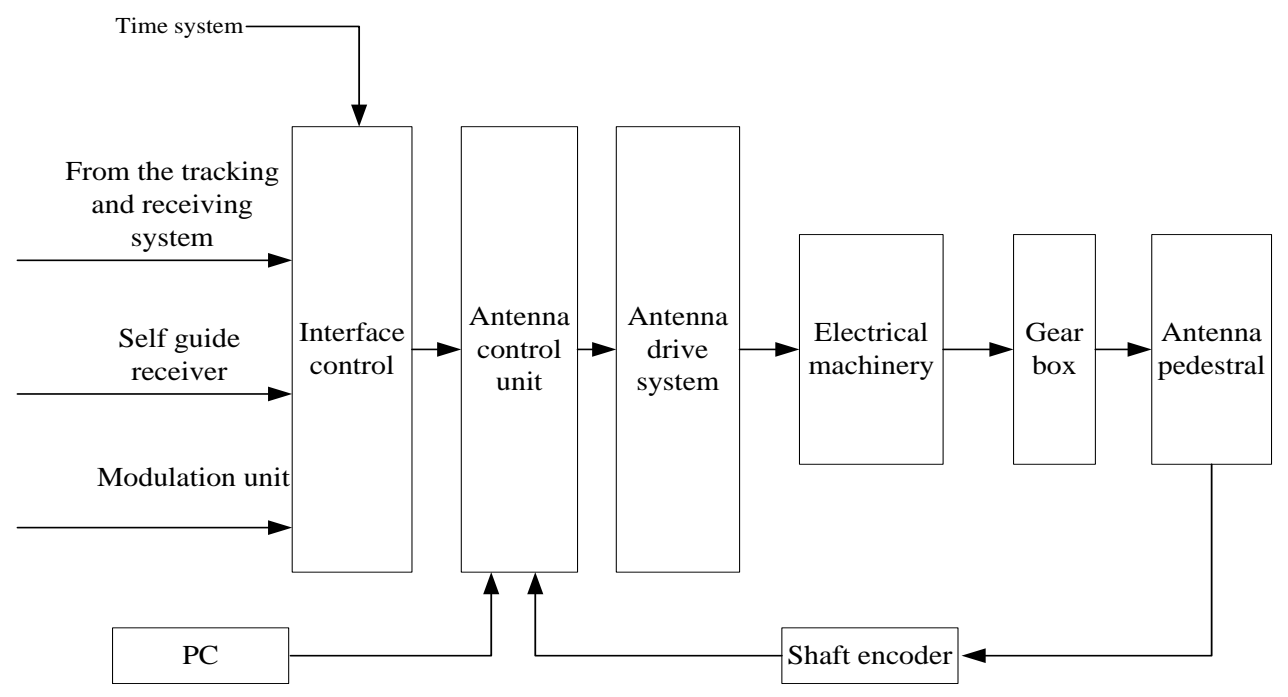

Figure 1. Radar antenna servo system diagram

As shown in Figure 1, radar servo system in general by the interface control, antenna control unit, drive unit, motor, gear box, antenna pedestal and the host computer and shaft encoder. Among them, the interface control part mainly completes and tracking receiver, guiding receiver communication, antenna control unit will be required for antenna control signal into the antenna driving unit of the input signal, and then the antenna driving unit drives the motor and gear box, drive complete antenna.

It can be seen from the above analysis, radar servo system is a mechatronics system, which has a high failure rate, test points, the fault location for short time, the time of fault diagnosis fault detection method is required for conventional far exceeds the maximum allowed equipment repair, rapid positioning method is needed to study fault.

\section{Point servo system optimization strategy based on particle swarm optimization algorithm}

From the above analysis we can see, fault diagnosis and location time is mainly due to more test points of radar servo system, and the fault location of all test points were measured to complete. If we can use some methods, the detection rate of a certain fault conditions reduce the required test points, it can reduce the time of fault diagnosis.

A. Servo system test point distribution

As shown in Figure 1, it can be seen that, the test point of radar servo system is mainly concentrated in the antenna control unit and antenna drive unit, on the security, fire control, artillery, reconnaissance radar was concluded radar servo system each part of test point distribution as shown in table 1.

Table1. Test points distribution of radar servo system

\begin{tabular}{ccccc}
\hline radar type & $\begin{array}{c}\text { Antenna control } \\
\text { unit }\end{array}$ & $\begin{array}{c}\text { Antenna drive } \\
\text { system }\end{array}$ & $\begin{array}{c}\text { Time control } \\
\text { unit }\end{array}$ & $\begin{array}{c}\text { Shaft } \\
\text { encoder }\end{array}$ \\
\hline Svrveillance radar & 15 & 15 & 23 & 19 \\
Fire-contrl radar & 18 & 14 & 15 & 17 \\
Coast defense radar & 9 & 8 & 11 & 8 \\
Artillery radar & 6 & 5 & 8 & 6 \\
\hline
\end{tabular}

Table 1 gives the distribution of each subsystem or unit testing different types of radar servo system, it is seen from table 1, test point radar servo system is mainly focused on the antenna 
control large warm antenna driving unit, if the two points system failure, will cause the fault diagnosis time longer, so fast fault location method based on the two subsystems.

\section{B. particle swarm optimization algorithm}

Particle swarm optimization algorithm is a stochastic global optimization technique proposed by reference [2] through the observation of the birds, fish and human social behavior, has the ability to find the global optimal solution in a high dimensional space, can solve large nonlinear, non differentiable and multi peak complex optimization problems, the realization of a simple, fast convergence speed, good stability, is widely applied in a plurality of methods of signal processing, image processing. The genetic algorithm with the widely used similar, PSO algorithm by the cooperation and competition among individuals, to achieve global optimal search. The PSO algorithm first generates a random particle in the feasible solution space, each particle as an initial solution, including position and velocity of two features. The merits of the position coordinates of particles by the cost function to obtain particle set value to measure, while its speed characteristics determine the direction of movement and displacement of the particle size of the next moment. In each optimization iteration, particle tracking through two kinds of "optimal" to update their position and speed of [3]: one is the optimal particle itself so obtained solutions, called individual extreme value, cognitive level represents the particle itself; the optimal second for all particles so far obtained solution, called the global extreme value, representative of social cognition

The standard particle swarm optimization algorithm for the [4] process is as follows:

1) The random initialization of each particle's speed and position, and the best $P$ individual set as current location, and the optimal individual groups as the current $\mathrm{P}$;

2) The speed and position of each particle in the D dimension of I were updated

3) In every generation of the evolution of each particle, calculation of the value of the fitness function;

4) If the particle is the current value of the fitness function is better than the optimal value of the history, current position to replace the historical best position of individual;

5) If the particle history optimal global optimum is better, then the global optimum is the best particle instead of [5];

6) If you haven't reached the end condition, to 2), otherwise the output $P$ and end. Characteristics of particle swarm algorithm is simple, easy to achieve, in various fields have been widely applied, based on this, this paper will introduce the process of the test point selection of radar servo system, in order to reduce the required test points to improve the efficiency of fault diagnosis, fault diagnosis.

\section{Construction and optimization objective function.}

Construct the objective function to consider the computational complexity of the algorithm, the difficulty of algorithm implementation and operation effects of the algorithm.

Fault diagnosis can accept the hypothesis of radar servo system fault rate is P0,the false alarm rate is $\mathrm{P} 1$, the time of fault diagnosis can accept is t0, then construct the objective function is as follows:

$$
J(x)=\min \left(\frac{p_{0}}{p_{1}}+t_{0}-m_{\mathrm{i}}\right)
$$

Among them, MI number ranges for test points, which ranges from [1, 20], 1 is the minimum required a test point, 20 is the maximum, i.e. on all test points tested to complete fault diagnosis.

The problem of solving the existing, on the following: first, select the number of particle swarm initialization, under normal circumstances, the more the number of particles, the more likely to converge to the global optimal solution, but the convergence speed will slow down, reduce the operating efficiency, algorithm instead, when selecting the number of particles less, though the convergence speed will be increased, but easy to converge to the local optimal solution, the above two factors make a compromise, selected 20 the number of particles in particle swarm as; second, re initializing particle rule selection, particle swarm algorithm, in a certain period of time after the particle re allocation optimization, in order to improve the convergence to the global optimum solution speed, In this paper, combined with the actual situation of radar servo system, determine 
the reinitialization of particle swarm optimization when half the total particle swarm optimization PSO entropy is more than.

Based on the above principle, experiment this paper selects antenna radar servo system control unit test point selection, to verify the validity of our algorithm.

\section{Simulation experiments}

Simulation results of a certain type of radar the antenna servo system control unit, using the method of fault injection simulation, and record the time of traditional fault diagnosis and test point algorithm based on particle swarm optimization fault diagnosis time, in contrast to the advantages and disadvantages of the two methods.

The antenna of a radar system control unit as shown in Figure 2.
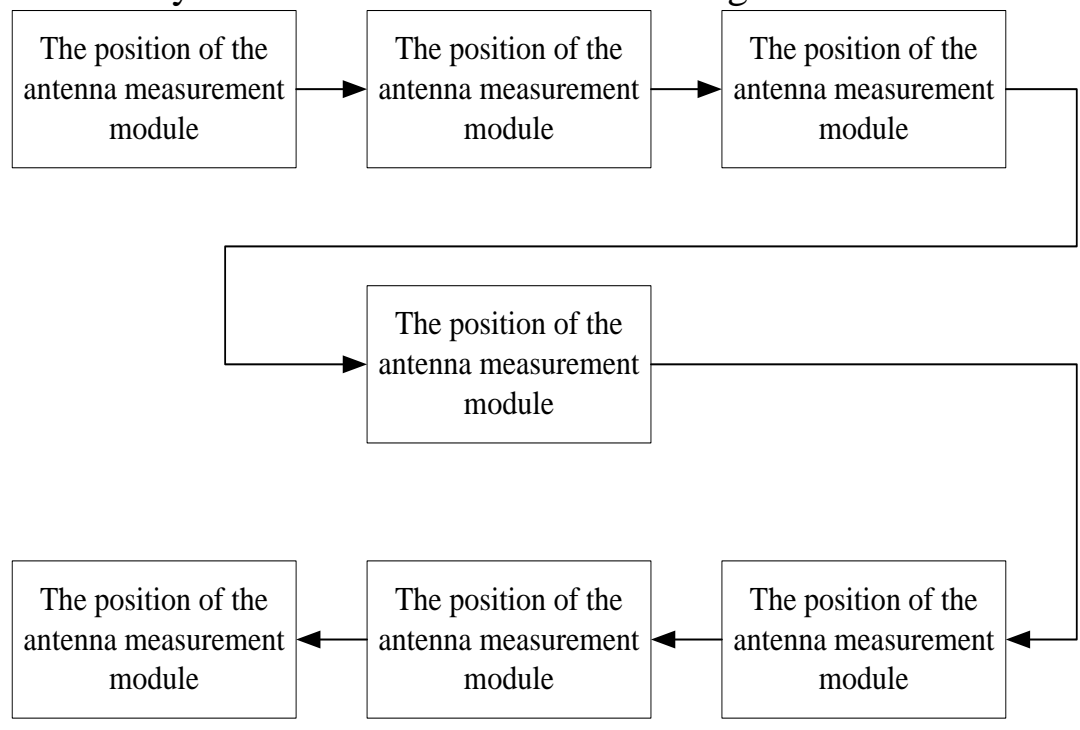

Figure 2. radar antenna control unit system diagram

As you can see from Figure 2, the type of radar antenna control unit by the antenna position measuring module, axial angle coding module, tracking error signal generation module. Module control unit antenna, test points, and the system is prone to failure, fault diagnosis time is long, therefore, implantation with fault, let the signal processing module of the control unit fault, and then use the ordinary method and intelligent optimization based fault diagnosis to the method of particle swarm, the diagnosis results as shown in table 2.

Table 2. Comparison of diagnosis results with different methods

\begin{tabular}{cccccc}
\hline $\begin{array}{c}\text { Fault } \\
\text { name }\end{array}$ & $\begin{array}{c}\text { Test point } \\
\text { number }\end{array}$ & $\begin{array}{c}\text { Request test } \\
\text { point number }\end{array}$ & $\begin{array}{c}\text { Fault diagnosis } \\
\text { rate }\end{array}$ & $\begin{array}{c}\text { False } \\
\text { alarm rate }\end{array}$ & Diagnosis method \\
\hline $\begin{array}{c}\text { Larger } \\
\text { error }\end{array}$ & 23 & 11 & $99 \%$ & $45 \%$ & Traditional method \\
$\begin{array}{c}\text { signal } \\
\text { Larger } \\
\text { error } \\
\text { signal }\end{array}$ & 16 & 8 & $96 \%$ & $45 \%$ & Traditional method \\
$\begin{array}{c}\text { Smaller } \\
\text { error } \\
\text { signal }\end{array}$ & 23 & 11 & $99 \%$ & $45 \%$ & New \\
$\begin{array}{c}\text { Smaller } \\
\text { error } \\
\text { signal }\end{array}$ & 16 & 7 & $98 \%$ & $45 \%$ & method \\
\hline
\end{tabular}

As you can see from Table 2, the type of radar antenna control unit by the antenna position measuring module, axial angle coding module, tracking error signal generation module. Module 
control unit antenna, test points, and the system is prone to failure, fault diagnosis time is long, therefore, implantation with fault, let the signal processing module of the control unit fault, and then use the ordinary method and intelligent optimization based fault diagnosis to the method of particle swarm, the diagnosis results as shown in Table 2.

\section{Conclusion}

Particle swarm optimization method because of its simple and easy to implement, and is not easy to fall into local optimum, has been widely used in many fields. This paper introduced into the fault diagnosis system of radar antenna servo system, the high rate of fault diagnosis and maintenance of the fault, lower false alarm rate, but shorten the diagnosis time, improve the efficiency of fault diagnosis. The method to the radar system fault diagnosis based on further research on this subject group.

\section{References}

[1]XI Haiyan. Application of MCS_96 MCU system in radar antenna servo system[J]. Communication and measurement contrl, 2010(28)10:126-131.

[2]KennedyJ, EBerhartR. Particle swarm optimization[C]// Proc. Of the IEEE International Conference on NeuralNet—works,1995:1942-1948.

[3]GAO Yanzhao, ZHAN Ronghui. Parameter estimation of KK distribution based on particle swarm optimization algorithm[J]. Systems Engineering and Electronics, 2013(35)12:2495-2500.

[4]ZHOU Lijun, PENG Wei, ZENG Xiaoqiang, et al. Dynamic Particle Swarm Optimization Based on Hybrid Variable[J].Computer Science, 2013(40)11:143-146.

[5]ZHU Hongfei, YANG Guang. Intelligent Test System Design Based on PXI Bus Module[J].Computer Measurement \& Control, 2013(21)11:2912-2914.

[6]MARTORELLA M, PALMER J, HOMER J, et al. On bistatic inverse synthetic aperture radar[J]. IEEE Transactions on Aerospace and Electronic Systems, 2007, 43(3):1125-1134..

[7]PALMER J, HOMER J, LONGSTAFF I D, et al. Radar target detecton using an emulated multistatic radar system[J]. IEEE Transactions on Aerospace and Electronic Systems, 2009, 41(4):1464-1472..

[8]PALMER J, HOMER J, MOJARRABI B. Improving on the monostatic radar cross section of targets by employing sea clutter to emulate a bistatic radar[C]. IEEE International Geoscience and Remote Sensing Symposium, Toulouse, France, July 21-25, 2003..

[9]JIN Tian, HUANG Xiao-tao, CHANG Wen-ge. Effect of high velocity target on range profile and correction method[J]. Systems Engineering and Electronics, 2011, (26)8: 1019-1022..

[10]FADILI M J, STARCK J L, MOUDDEN Y, et al. Image decomposition and separation using sparse representations: an overview[J]. Proceedings of the IEEE, 2010, 98(6):983-994.

[11]HE Huiwen, PENG Cheng, ZHAO Mei, et al. A Study on Frequency Domain Identification and Control Method in Radar Servo System[J]. Modern Radar, 2013,35(9):53-56.

[12]SUN Wenxin, MU Huaping, GAO Fengli. Particle swarm optimization based on self-adaptive population structure[J]. CAAI Transactions on Intelligent Systems, 2013,8(4):372-375;

[13]LI Xuefa, ZHOU Yinghong, YANG wei. Projection Pursuit Evaluation Model for Water Quality Based on the Particle Swarm Optimization Algorithm[J]. Hubei Agriculture Science, 2013,(52)19:4627-4630. 